\title{
TAXONOMÍA FORMAL
}

\author{
Jesús MOSTERÍN
}

\section{CLASIFICAR}

Una de las actividades científicas más frecuentes es la que consiste en clasificar ${ }^{\dagger}$ los individuos de un ámbito determinado, de tal modo que podamos hablar, pensar y formular leyes o hipótesis sobre ellos con más facilidad. Cuando nos ponemos a clasificar un dominio de objetos, no consideramos terminada nuestra tarea hasta que la clasificación o colección de clases introducidas los abarca a todos. Esto puede precisarse diciendo que el resultado de clasificar un conjunto $A$ ha de constituir un recubrimiento de $A$.

Un recubrimiento de $A$ es una familia de subconjuntos no vacios de $A$ tal que la unión de todos ellos es idéntica a $A$. Formalmente,

\section{1. $G$ es un recubrimiento $\operatorname{de} A$}

$\Leftrightarrow G \subset \mathscr{P} A \wedge \varnothing \notin G \wedge \cup G=A$.

Las clases que constituyen una clasificación pueden solaparse, pueden ser solapantes. Por ejemplo, la clasificación de los humanes por nacionalidades es solapante, pues hay individuos con doble nacionalidad. La clasificación de los estudiantes de una universidad por la facultad en que están matriculados puede ser solapante, pues algunos alumnos pueden estar matriculados en más de una facultad. La clasificación ecológica de los animales por el tipo de ecosistemas en que se encuentran es solapante, pues algunos animales moran en ecosistemas de diverso tipo. Frente a esta clasificación ecológica la clasificación sistemática de los animales no admite solapamientos, sino que pretende clasificarlos de tal modo que un mismo animal no pueda estar en dos clases distintas (del mismo nivel), es decir, pretende ser una clasificación no-solapante. La clasificación de los átomos en elementos químicos y la clasificación de los humanes por su año de nacimiento también son no-solapantes. El resultado de clasi* ficar $A$ no-solapantemente constituye no sólo un recubrimiento de $A$, sino incluso una partición de $A$.

Una partición de $A$ es un recubrimiento de $A$ cuyas clases son todas disjuntas entre sí, es decir, sin elementos comunes. Formalmente,

\section{2. $G$ es una partición de $A$}

$\Leftrightarrow G \subset \mathscr{P} A \wedge \varnothing \notin G \vee \cup G=A \wedge \forall X Y$ $(X \in G \wedge Y \in G \wedge X \neq Y \Rightarrow X \cap Y=\varnothing)$
Las clasificaciones más importantes científicamente son las no-solapantes, y a cllas nos limitaremos aquí. En lo sucesivo, siempre que hablemos de clasificaciones, queremos decir clasificaciones no-solapantes.

Suele llamarse clasificación tanto a la actividad de clasificar como al resultado de esa actividad, la partición. Esta ambigüedad es inofensiva. Más grave es que a veces se utiliza la misma palabra clasificación para referirse a dos actividades o procesos totalmente distintos: la clasificación de un dominio de individuos en clases, por un lado, y el diagnóstico o identificación, ${ }^{2} \mathrm{dc}$ uno de esos individuos como perteneciente a una de esas clases previamente establecidas, por otro. La primera constituye una actividad científica creativa, mientras que la segunda es una mera práctica. Esta distinción es paralela a la que puede establecerse entre metrización (introducción de una magnitud métrica en un campo previamente cualitativo) y medición (determinación del valor concreto de esa magnitud para un individuo determinado). No puede haber medición sin metrización previa, como no puede haber diagnóstico sin previa clasificación.

El médico diagnostica la dolencia concreta que padece el paciente en un momento dado interpretándola o reconociéndola como (elemento de) una enfermedad determinada, lo cual presupone una previa clasificación de las dolencias concretas en enfermedades. F.l naturalista de campo, o el aficionado provisto de una guía de campo, diagnostica o identifica una planta o un pájaro que tiene ante sus ojos como perteneciente a una cierta clase de una clasificación previamente establecida. A las clases establecidas por una clasificación les llamamos taxones. En este sentido, una enfermedad (clase de dolencias) o un elemento químico (clase de átomos) o una especie biológica (clase de organismos) es un taxón y, por tanto, el diagnóstico es el proceso que asigna un individuo de un dominio determinado al taxón que le corresponde (al que pertenece).

El diagnóstico es, pues, una función que a cada individuo de un dominio determinado asigna el taxón de una clasificación o partición dada, ese dominio al que el individuo en cuestión pertenece. Puesto que todo individuo pertenece a un taxón y sólo a uno de una partición dada, el taxón al que ese individuo pertenece está unívocamente determinado. la función $d_{Q}$ diagnóstica 
depende de la partición dada $Q$. Sea $Q$ una partición de $A . d_{Q}: \mathrm{A} \rightarrow \mathrm{Q}$. Definimos:

\section{3. $d_{Q}=\{(a, Z) \mid a \in A \wedge Z \in Q \wedge a \in Z\}$}

La tarea de diagnosticar o identificar $a \in A$ respecto a la partición $Q$ de $A$ significa calcular el valor $d_{\mathrm{Q}}(a)$. Para ello a veces nos servimos de la observación de los síntomas observables de a y los comparamos con los síndromes (conjuntos de síntomas característicos) de los diversos taxones. Otras veces hemos de considerar ciertas relaciones en que $a$ está o no está con otros individuos, etc.

La nomenclatura es la asignación de nombres convenidos por la comunidad científica a los diversos taxones distinguidos en la clasificación. Pero aquí no vamos a ocuparnos del diagnóstico, ni de la nomenclatura, sino sólo del resultado directo de la clasificación: las particiones.

\section{PARTICIONES Y RELACIONES DE EQUIVALENCIA}

2.1. Como es sabido, hay una estrecha correlación entre las particiones y las relaciones de equivalencia, y aquí mismo usaremos más adelante una cierta relación de equivalencia para definir una partición (la fusión de dos particiones dadas) que nos interesa caracterizar.

Una relación de equivalencia en $\mathrm{A}$ es una relación $R \subset A \times A$, que es reflexiva, simétrica y transitiva.

Por ejemplo, la identidad es una relación de equivalencia en cualquier conjunto. El paralelismo es una relación de equivalencia entre rectas. El tener el mismo número de protones en el núcleo es una relación de equivalencia entre átomos.

Si $R$ es una relación de equivalencia en $A$ y $x$ en un miembro de $A$, entonces el conjunto de todos los miembros de $A$ que están en la relación $R$ con $x$ se llama la clase de equivalencia (respecto a $R$ ) de $x$, simbolizada como $x_{R}$. $x$ mismo es un representante de $x_{R}$. Definimos:

\section{2. $x_{R}=\{y \in \mathrm{A} \mid y R x\}$}

De aquí se sigue como corolario que dos clases de equivalencia $x_{R}$ y $z_{R}$ son idénticas si y sólo si sus representantes $x$ y $z$ están entre sí en la relación de equivalencia $R$.

\section{3. $\forall x z \in A\left(x_{R}=z_{R} \Leftrightarrow x R z\right)$}

Llamemos $\operatorname{Part}_{A}$ al conjunto de todas las particiones de $A$. Y llamemos $\mathrm{Eq}_{A}$ al conjunto de todas las relaciones de equivalencia en $A$. La correlación aludida anteriormente consiste en que cada partición de $A$ determina una cierta relación de equivalencia en $A$, y cada relación de equivalencia en $A$ determina una cierta partición de $A$.

Sea $Q$ una partición de $A$. La relación de equivalencia $R_{Q}$ inducida o determinada por $Q$ es la relación en que están dos individuos de $A$ si y sólo si ambos pertenecen al mismo Q-taxón. Definimos:

$$
\text { 2.4. } R_{Q}=\left\{(x, y) \in A^{2} \mid d_{Q}(x)=d_{Q}(y)\right\}
$$

Fácilmente se comprueba que $R_{Q}$ es una relación de equivalencia.

Sea $R$ una relación de equivalencia en $A$. La partición $A / R$ de $A$ inducida o determinada por $R$ es el llamado espacio cociente de $A$ respecto a $R$, es decir, la clase de todas las relaciones de equivalencia de $R$. Definimos:

$$
\text { 2.5. } A / R=\left\{x_{R} \mid x \in A\right\}
$$

Fácilmente se comprueba que $A / R$ es una partición de $A$.

Precisamente una partición cualquicra de $A$ coincide con el espacio cociente de $A$ respecto a la relación de equivalencia inducida por esa partición. En resumen:

\section{6. $R \in \mathrm{E}_{\mathrm{q}_{A}} \Rightarrow A / R \in \mathrm{Part}_{A}$ \\ 2.7. $Q \in \operatorname{Part}_{A} \Rightarrow R_{Q} \in \mathrm{Eq}_{A}$ \\ 2.8. $Q \in \operatorname{Part}_{A} \Rightarrow Q=A / R_{\mathrm{Q}}$}

Con frecuencia se introducen las particiones mediante relaciones de equivalencia. La partición de las rectas del plano en direcciones es la partición inducida por la relación de equivalencia de paralelismo, es decir el conjunto cociente de las rectas por el paralelismo. La partición de los átomos en elementos químicos es la partición inducida por la relación de equivalencia de tener igual número de protones en el núcleo, es decir, el conjunto cociente de los átomos por la igualdad 
del número de protones. La partición de los fonos de una lengua en fonemas es la partición inducida por la relación de equivalencia de sustituibilidad salva significatione, es decir, el conjunto cociente de los fonos por la sustituibilidad salva significatione.

Llamemos $l_{A}$ es la identidad restringida a $A$, es decir, $l_{A}=\left\{(x, y) \in A^{2} \mid x=y\right\}, y$ llamamos $A^{2}$ a la clase de todos los pares de elementos de $A$, es $\operatorname{decir}, A^{2}=A \times A$.

Como fácilmente se comprueba, $I_{A}$ es no sólo una relación de equivalencia en $A$, sino incluso la mínima relación de equivalencia en $A$, en el sentido de que está incluida en todas las demás. $A^{2}$, por el contrario, no sólo es también una relación de equivalencia en $A$, sino que además es la máxima, en el sentido de que todas las demás están incluidas en ella. En resumen:

2.9. $I_{A} \in \mathrm{Eq}_{A}$

2.10. $l_{A}=\cap \mathrm{\cap Eq} q_{A}$

2.11. para toda R: $\left(R \in \mathrm{Eq}_{A} \Rightarrow I_{A} \subset R\right)$

2.12. $A^{2} \in \mathrm{Eq}_{A}$

2.13. $A^{2}=U \mathrm{Eq} A$

2.14. para toda $\mathrm{R}:\left(R \in \mathrm{Eq}_{A} \Rightarrow R \subset A^{2}\right)$

2.15. $\left\langle\mathrm{Eq}_{A}, C_{n}\right\rangle$ es una ordenación parcial, con mínimo, $I_{A}, y$ máximo, $A^{2}$.

\section{LA RELACION DE MAYOR O IGUAL FINURA}

Un dominio $A$ de individuos puede clasificarse o partirse de muy diversas maneras, tantas como elementos posee Part $_{A}$. Así por ejemplo, los animales pueden clasificarse geográficamente, según el continente u océano en que viven, o ecológicamente, según el tipo de biotipo que habitan, o sistemáticamente por especies, o sistemáticamente por órdenes, etc. Unas clasificaciones o parti* ciones son a veces más finas que otras, pero con frecuencia son incomparables entre sí. La clasificación geográfica de los animales es incomparable con su clasificación sistemática en especies, pero esta última es comparable con su clasificación sistemática en órdenes y resulta más fina que ella.

Una partición es más (o igual de) fina que otra cuando hace todas las distinciones que esa otra hace, y quizás todavía algunas más. Más precisa mente, una partición $P$ es más (o igual de) fina que $Q$ si y sólo si cada taxón de $P$ está incluido en un taxón de $Q$. Cada especie está incluida en un género y en un orden. Por eso la clasificación de los animales en especies es más fina que la clasificación en géneros o en órdenes. Simbolicemos la relación de mayor o igual finura entre particiones mediante «\$», Sean $P$ y $Q$ particiones de $A$. Dcfinimos:

\section{1. $P \leqslant Q \Rightarrow \forall X \in P \exists Z \in Q(X \subset Z)$}

Otra condición equivalente (no entre recubrimientos en general, pero sí entre particiones) es la de que cada taxón de la primera partición esté incluido en o sea disjunto con cada taxón de la segunda.

\section{2. $P \leqslant Q \Rightarrow \forall X \in P \forall Z \in Q$ \\ $(X \subset Z \vee X \cap Z=\varnothing)$}

Como fácilmente se aprecia, la relación $\leqslant$ de mayor o igual finura entre particiones de $A$ es reflexiva, antisimétrica y transitiva, es decir, es una relación de orden parcial en Part A $_{A}$. Esta ordenación parcial tiene un mínimo, el conjunto de todas las clases unitarias o singletones de $A$, al que simbolizaremos por $\mathrm{Sng}_{A}$ y tiene un máximo, $\{A\}$. En efecto, Sng ${ }_{A}$ no sólo es una partición de $A$, sino que además es más fina que cualquier otra partición de $A$. Y $\{A\}$ no sólo es una partición de $A$, sino que es la más grosera de todas las particiones de $A$.

3.3. $\operatorname{Sng}_{A}=\{\{x\} \mid x \in A\}$

3.4. $\forall Q \in \operatorname{Part}_{A}:$ Sng $_{A} \leqslant Q$

3.5. $\forall Q \in \operatorname{Part}_{A}: Q \leqslant\{A\}$

En resumen, $\left\langle\mathrm{Part}_{A}, \leqslant\right.$ es una ordenación parcial con mínimo, $\mathrm{Sng}_{A}, y$ máximo, $\{A\}$.

\section{JERARQUIAS TAXONOMICAS}

Un mismo dominio de individuos puede clasificarse de muy diversas maneras, dando lugar a distintas particiones del mismo. Así, podemos clasificar los minerales por su color, o por su estructura cristalina, o por su composición química, o por el lugar en que se encuentran, etc. Todas estas clasificaciones son independientes unas de otras y son incomparables entre sí en cuanto a finura. No forman una jerarquía taxonómica. 
Otras veces sin embargo nos encontramos con clasificaciones interdependientes, cuyas particiones son comparables entre sí. Así podemos clasificar las dolencias que nos aquejan en hereditarias y adquiridas. Más finamente podemos clasificar las adquiridas en traumáticas, degenerativas $\mathrm{e}$ infecciosas. Todavía más finamente podemos clasificar las infecciosas en víricas, bacterianas, fúngicas, etc. Podemos seguir afinando más y clasificar las bacterianas en producidas por bacilos, por estreptococos, etc. Y aún más finamente podemos clasificar las dolencias bacterianas producidas por bacilos en enfermedades como el tétanos, la tuberculosis, etc. Todas estas clasificaciones son comparables entre sí en cuanto a finura. Forman una jerarquía taxonómica. Todos los individuos (en este caso, las dolencias concretas) que sean miembros de un taxón de la partición más fina (cn este caso, la partición en enfermedades), serán también miembros de un mismo taxón en cada una de las otras clasificaciones. Todas las dolencias concretas que sean casos de tuberculosis serán producidos por bacilos, de origen bacteriano, infecciosas y adquiridas.

La mayoría de los subconjuntos de $\operatorname{Part}_{A}$, es decir, la mayoría de los conjuntos de particiones de $A$ contienen particiones incomparables entre sí en cuanto a finura. Si ocurre que un conjunto $H$ de particiones de $A$ sólo conticne particiones comparables entre sí en cuanto a finura, diremos que $H$ constituye una jerarquía taxonómica sobre $A$.

\section{1. $H$ es una jerarquía taxonómica sobre $A \Leftrightarrow \Rightarrow$ $H \subset \operatorname{Part}_{A} \wedge \forall X Y \in H(X \leqslant Y \vee Y \leqslant X)$}

Habiamos visto en 3 que $\left(\operatorname{Part}_{\mathcal{A}}\right.$, $\leqslant$ es una ordenación parcial. Un subsistema de $\left(\operatorname{Part}_{A} \leqslant\right.$ que constituya una ordenación total (o lineal o cadena, según la terminología que se prefiera) constituye siempre una jerarquía taxonómica sobre $A$. Por eso una definición alternativa de jerarquía taxonómica es:

\section{2. $H$ es una jerarquía taxonómica sobre} $A \Leftrightarrow H \subset$ art $_{A} \wedge\langle H, \leqslant$ es una ordenación total

En el contexto de una jerarquía taxonómica $H$, las diversas particiones que forman $H$ se suelen llamar categorías de $H$. Así los individuos del dominio básico $A$ son miembros de los taxones de diversas particiones o categorías. Los taxones mismos son miembros de las particiones o categorías. Y las particiones o categorías son miembros de la jerarquía taxonómica.

Las jerarquías taxonómicas más conocidas son las usadas en la biología. La llamada jerarquía taxonómica linneana consta de siete categorías: especie, género, familia, orden, clase, phylum y reino, que constituyen otras tantas particiones del dominio de los organismos. Así, un organismo concreto, por ejemplo, esta abeja que pasa ahora zumbando por aquí, pertenece a la especic Mellifera, al género Apis, a la familia Apidae, al orden Hymenoptera, a la clase Insecta, al phylum Arthropoda y al reino Animalia. Cada uno de los taxones citados está incluido en todos los siguientes. Cuando decimos que esta abeja pertence por ejemplo al género Apis, queremos decir que pertence al taxón $A p i s$, cl cual, a su vez, pertenece a la categoría género (o, si se prefiere, en latín, genus). Esta abeja es un individuo que es miembro del taxón Apis. El taxón Apis es miembro de la categoría género. La categoría género es miembo de la jerarquía taxonómica linneana.

A partir de la jerarquía linneana se obtienen a veces otras jerarquías taxonómicas más amplias añadiendo nuevas categorías, como tribu (entre género y familia) o cohorte (entre orden y clase), o como las formadas con los prefijos super y sub (superfamilia, subfamilia, etc.).

Dada una jerarquía taxonómica $H$ cada categoria de esa jerarquía tiene un cierto rango o nivel. Puesto que todas las categorías o particiones de $H$ son comparables entre sí, podemos ordenarlas de tal modo que la más fina aparezca en primer lugar (tenga rango 1), la siguiente más fina tenga rango 2 , etc., hasta llegar a la menos fina, que tendrá máximo rango o nivel. Así, en la jerarquía taxonómica linneana la categoría especie tiene rango 1, la categoría género tiene rango 2, la categoría familia tiene rango 3 , la categoría orden tiene rango 4, la categoría clase tiene rango 5 , la categoría phylum tiene rango 6 y la categoría reino tiene rango 7.

Sea $H$ una jerarquía taxonómica que conste de $n$ particiones o categorías. Puesto que $(H, \leqslant)$ es una ordenación total, hay un isomorfismo de $\langle H, \leqslant$ con $\{(1,2, \ldots \mathrm{n}\}, \leqslant\rangle$ es decir, una función biyectiva $f: H \rightarrow\{1,2, \ldots \mathrm{n}\}$ tal que para cada $X, Y \in H$ : 


$$
X \leqslant Y \Leftrightarrow f(X) \leqslant f(Y)
$$

Esta función está univocamente determinada y constituye el rango. Para cualquier $X \in H$ :

\section{3. $\operatorname{rango}(X)=f(X)$}

Naturalmente esta definición sólo vale para jerarquías finitas, es decir, con un número finito de particiones o categorías, que son las que se usan en la ciencia. Si quisieran considerarse también jerarquías infinitas bastaría con tomar segmentos iniciales cualesquiera del conjunto $\Omega$ de todas los ordinales, en vez de segmentos iniciales de $\mathbb{N}$, como hemos hecho aquí.

El rango de una categoría o partición de una jerarquía taxonómica es un número natural. Cuanto mayor es el rango tanto menor la finura de la partición. Para cualesquiera particiones $X$, $Y$ de una jerarquía $H$ :

\section{4. $X \leqslant Y \Leftrightarrow$ rango $(X) \leqslant$ rango $(Y)$}

También se suele decir que un taxón tiene rango, a saber, el rango de la categoría a la que ese taxón pertence. Así, en la jerarquía taxonómica linneana el género Apis tiene rango 2, es decir, el género Apis pertenece a la categoría género, que es la que propiamente tiene rango 2.

\section{LA PARADOJA DE GREGG}

Los biólogos clasifican los organismos en taxones de diverso rango. Normalmente los taxones de rango superior incluyen varios taxones de cada rango inferior $y$, por ello, tienen más miembros que ellos y son extensionalmente distintos de ellos. Así, por ejemplo, la clase Insecta incluye muchos otros órdenes, además de Hymenoptera; el orden Hymenoptera incluye otras muchas familias, además de Apidae. La familia Apidae incluye otros géneros, además de Apis, etc. Asi, pues, el caso normal consiste en que un taxón de cierto nivel es un subconjunto propio de otro taxón de nivel superior, pero no coincide con él. Estos taxones normales, que incluyen varios taxones de nivel inferior dado, se llaman taxones politípicos. Sin embargo, no todos los taxones son politípicos, también los hay monotípicos. Veamos algunos ejemplos.
Los botánicos clasifican las plantas del modo ya indicado en especies, géneros, familias, órdenes, etc. Así los ginkgos, los conocidos árboles procedentes de la China con hoja en forma de abanico pertencen a la especie Ginkgo biloba, al género Ginkgo, a la familia Ginkgoaceae y el orden Ginkgoales. Pero todos esos taxones, de diferente rango, contienen exactamente los mismos individuos: los ginkgos. El orden Ginkgoales incluye una sola familia, que incluye un solo género, que posee una sola especie. Todos esos taxones son monotípicos.

Los ornitólogos clasifican las aves dentro de las categorías de la jerarquía linneana. Los kiwis, primitivas aves sin alas típicas de Nueva Zelanda, son los únicos animales pertenecientes al género Apteryx, a la familia Apterygidae y al orden $A p$ terygiformes, que, por tanto, son taxones monotípicos. El orden Apterygiformes contiene una sola familia, Apterygidae, que a su vez incluye un solo género, Apteryx el cual, sin embargo, se subdivide en 3 especies distintas.

Los ornitorrincos son unos primitivos mamíferos australianos y los zoólogos los agrupan en una sola especie, Ornithorbynchus anatinus, que es la única de que consta el género Ornithorbyncbus, que a su vez es el único incluido en la familia de los Ornithorhynchidae. Los tres taxones mencionados son por tanto monotípicos.

Los oricteropos o cerdos hormigueros son unos mamíferos africanos de largo morro cilindrico y poderosas patas delanteras que les sirven para excavar la tierra. Los zoólogos los clasifican en una sola especie, Orycteropus afer, que es la única de que consta el género Orycteropus, que a su vez es el único que está incluido èn la familia Orycteropodidae y en el orden Tubulidentata. Todos estos taxones son también monotípicos.

Si los taxones de la clasificación biológica son conjuntos, entonces los taxones monotípicos (que tienen los mismos elementos) han de ser idénticos, pues dos conjuntos con los mismos elementos son el mismo conjunto. Pcro los biólogos sistemáticos, que establecen las clasificaciones, piensan que una especie es siempre algo muy distinto de una familia, por ejemplo. Por tanto, aunque una familia y una especie tengan los mismos elementos (organismos), serán taxones distintos. El primero que se dio cuenta de esta dificultad fue John R. Gregg, y desde entonces se conoce como la paradoja de Gregg. 
Desde 1954 en que Gregg ${ }^{3}$ llamó la atención sobre la paradoja que lleva su nombre, se han propuesto diversos intentos de solución.

En 1957 Parker-Rhodes ${ }^{4}$ propuso definir un taxón superior no como un conjunto de individuos (es decir, de organismos), sino como el conjunto de sus taxones inmediatamente inferio res. Con esto se soluciona la paradoja de Gregg, pero se aparta uno tambićn de la noción intuitiva de taxón. Así, un taxón de clase ya no sería un conjunto de organismos, sino un conjunto de órdenes. No podríamos seguir diciendo que este gato es un mamífero. La solución resulta insatis" factoria.

En 1964 A. Sklar ${ }^{5}$ propuso añadir a las $n$ particiones de una jerarquía $H n$ conjuntos (disjuntos con $U H$, por ejemplo, conjuntos de números naturales) $G_{1}, \ldots G_{n}$, tales que para cada $i, j$ $(1 \leqslant \mathrm{i}<\mathrm{j} \leqslant \mathrm{n}): G_{\mathrm{i}} \subset G_{\mathrm{j}}$, pero $G_{\mathrm{j}} \not \subset G_{\mathrm{i}}$, de tal modo que cada taxón $X$ de rango $k$ deviniese $X \cup G_{\mathrm{k}}$. Así (para retomar nuestro último ejemplo de taxones monotípicos), la especie Orycteropus afer devendria \{orictepopos\} $\cup\{1\}$, el nuevo género Oryteropus sería \{oricteropos\} $\cup\{1,2\}$, la nueva familia Oryteropodidae sería \{oricteropos $\} \cup\{1,2,3\}$, y el orden $T u b u l i-$ dentata sería (oricteropos\} $\cup\{1,2,3,4\}$. Con esto se soluciona la paradoja de Gregg, pues cada taxón es distinto de sus correspondientes taxones superiores y está incluido en ellos. Se trata de un truco técnico formalmente correcto, pues se conservan las relaciones de pertenencia de los organismos a los taxones (en este sentido es un pro* greso respecto a la solución de Parker-Rhodes) y las de inclusión entre taxones, al tiempo que se distinguen los taxones monotípicos de distinto rango (que se diferencian ahora por poseer algún número más o menos). Pero resulta artificioso concebir los taxones como conjuntos de algo más que de organismos, y resulta insatisfactorio decir que el número 1 es un cerdo hormiguero.

Fn 1966 R. C. Buck y D. L. Hull ${ }^{6}$ propusieron definir intensionalmente, mediante listas de propiedades, los taxones, de tal modo que se exigieran más propiedades para la pertenencia a los taxones de menor rango. Pero aquí hay una cierta confusión, pues los taxones siguen siendo considerados como conjuntos, es decir, como entidades extensionales. $Y$ dos conjuntos que tienen los mismos clementos son idénticos, cualquicra que sea el camino que sigamos para definirlos.
En 1969 propuso N. Jardine ${ }^{7}$ la primera solución satisfactoria de la paradoja de Gregg, que consistía en definir un taxón $T$ como un par ordenado $(D, r)$, donde $D$ es la extensión del taxón y $r$ es su rango. Así, aunque $T_{1}, T_{2}$ y $T_{3}$ sean taxones monotípicos (es decir, de igual extensión D) de distinto rango (es decir, de rango $r_{1}, r_{2}, r_{3}$, donde $r_{1} \neq r_{2} \neq r_{3}$ ), sin embargo son distintos taxones, pues $\left(D, r_{1}\right\rangle \neq\left\langle D, r_{2}\right\rangle \neq\left\langle D, r_{3}\right\rangle$.

Desde un punto de vista intuitivo lo más satisfactorio es considerar los taxones como conjuntos de organismos. El problema de Gregg puede resolverse (o, mejor dicho, disolverse) por cl trivial expendiente de distinguir los taxones a secas, que serán menos conjuntos de organismos, de los taxones jerarquizados, que serán pares ordenados de taxones a secas y rangos. Por tanto dos taxones monotípicos coinciden en cuanto taxones a secas (son el mismo conjunto dc organismos), pero difieren en cuanto taxones jerarquizados (pues poseen rango distinto). $\mathrm{Y}$ el que los consideremos de un modo u otro depende de nosotros, no de cllos.

\section{SUPERPOSICION DE PARTYCIONLS}

Frecuentemente obtenemos nuevas $\mathrm{c}$ intcresantes particiones superponicndo dos particiones que ya teniamos. la nueva partición así obtenida es más fina que ambas y recoge todas las distinciones hechas por cualquiera de ellas. Si $Q$ y $G$ son particiones de $A$, una manera usual de representar la superposición de $Q$ y $G$ consiste en dibujar una tabla cuyas filas correspondan a los taxones de $Q$ y cuyas columnas correspondan a los taxones de $G$. Los cuadros o casillas de la tabla representan entonces la nueva partición, que es la superposición de las dos anteriores. Por ejemplo, sea $A=\{0,1,2,3,4,5,6,7,8,9\}, Q=\left\{Q_{1}, Q_{2}\right\}$, donde $Q_{1}=\{0,1,2,3,4\}$ y $Q_{2}=\{5,6,7,8,9\}$, y $G=\left\{G_{1}, G_{2}, G_{3}, G_{4}\right\}$, donde $G_{1}=\{0\}$, $G_{2}=\{1,3,5,7,9\}, G_{3}=\{2,4\}$, y $G_{4}=\{6,8\}$. La tabla

G

\begin{tabular}{cc|c|c|c|c|} 
& $G_{1}$ & $G_{2}$ & $G_{3}$ & $G_{4}$ \\
\hline \multirow{2}{*}{$a_{1}$} & 0 & 1,3 & 2,4 & \\
\hline$O_{2}$ & & $5,7,9$ & & 6,8 \\
\hline
\end{tabular}


represenca la superposición de $Q$ y $G$. Esta superposición, $\{\{0\},\{1,3\},\{2,4\},\{5,7,9\},\{6,8\}\}$, es ella misma una nueva partición de $A$. Las casiIlas vacías de la tabla no forman parte de la superposición de $Q$ y $G$, pues queremos que ésta sea una partición y una partición es uná colección de clases no vacías.

La superposición de particiones para la producción de nuevas particiones ocurre en casi todas las ciencias, aunque hasta ahora (que yo sepa) no ha sido analizada. En fonologia, por ejemplo, la partición de las consonantes por su punto de articulación (en labiales, labiodentales, interdentales, alveolares, etc.) se superpone con frecuencia con la partición de las consonantes por su modo de articulación (en oclusivas, fricativas, africadas, vibrantes, etc.) para sí producir una nueva partición o clasificación de las consonantes, que es más fina y más informativa que cualquiera de las otras dos, tomadas por separado. No todos los ejemplos son tan triviales como éste. Uno de los más potentes resultados teóricos de la química, la tabla periódica de los elementos, es la partición de los elementos químicos que resulta de la superposición de la partición de los elementos químicos en grupos (en gases inertes, metales alcalinos, etc.), y de la partición de los elementos químicos en periodos. El hecho de que la mayoría de los taxones de la partición resultante contienen un solo miembro, mientras que dos de ellos tienen quince, complica la representación gráfica de la tabla, pero es evidente que se trata de una superposición de dos particiones dadas. Otro ejemplo teóricamente fecundo de superposición de particiones, esta vez provenien. te de la astronomía, está constituido por el diagrama de Hertzsprung-Russell, que es la partición de las estrellas que resulta de la superposición de la partición de las estrellas en clases espectrales y de la partición de las estrellas en magnitudes absolutas.

La superposición de dos particiones $P$ y $Q$ del mismo dominio $A$, que simbolizaremos mediantc $P \otimes Q$, consiste en la clase de todas las intersecciones no vacías de taxones de $P$ con taxones de $Q$. La operación $\otimes$ de superposición se define así:

$$
\begin{aligned}
& \text { 6.1. } P \otimes Q=\{Z \mid \exists X \in P \exists Y \in Q \\
& (Z=X \cap Y \wedge Z \neq \varnothing)\}
\end{aligned}
$$

A partir de esta definición se puede probar que la superposición de dos particiones de $A$ es siempre a su vez una partición de $A$, que $\otimes$ conduce de particiones de $A$ a particiones de $A$.

\section{2. $\otimes: \operatorname{Part}_{A} \times \operatorname{Part}_{A} \rightarrow \operatorname{Part}_{A}$}

Prueba. Sean $P$ y $Q$ particiones de $A$. Hemos de probar que $P \otimes Q$ es también una partición de $A$, lo cual a su vez nos obliga a pasar revista a las 4 condiciones de la definición de partición. (1) $P \otimes Q \subset \mathscr{P A}$. En efecto, cada elemento de $P \otimes Q$ es la intersección de dos subconjuntos de $A$ y, por tanto, es él mismo su subconjunto de $A$. (2) $\varnothing \notin P \otimes Q$, por definición de $P \otimes Q$. (3) $\cup(P \otimes Q)=A$. Sea $a \in U(P \otimes Q)$. Para algún $Z \in P \otimes Q, a \in Z$. Para algún $X \in P$ y algún $Y \in Q, Z=X \cap Y$. Luego $a \in X \cap Y, a \in X, y$ (puesto que $X \subset A$, ya que $X \in P$ y $P$ es una partición de $A) a \in A$. Por tanto $\cup(P \otimes Q) \subset A$. Sea ahora $b \in A$. Puesto que $P$ y $Q$ son particiones de $A, b \in d_{p}(b)$ y $b \in d_{Q}(b)$, por tanto $b \in d_{P}(b) \cap d_{Q}$ (b) y por consiguiente, para algún $X \in P$ (a saber, $\left.d_{P}(b)\right)$ y algún $Y \in Q$ (a saber, $\left.d_{Q}(b)\right) b \in X \cap Y$. Pero este $X \cap Y$, que no es vacío, pues tiene $b$ como elemento, es un miembro de $P \otimes Q$, por definición de $P \otimes Q$. Luego $b \in \mathrm{U}(P \otimes Q)$. Por tanto $A \subset \cup(P \otimes Q)$. Con lo que queda probado que $U(P \otimes Q)=A$. (4) Si $X \in P \otimes Q$, $Y \in P \otimes Q$ y $X \neq Y$ entonces $X \cap Y=\varnothing$. Sca $X \in P \otimes Q$ y $Y \in P \otimes Q$. Entonces habrá $X_{1}$, $Y_{1} \in P$ y $X_{2}, Y_{2} \in Q$, tales que $X=X_{1} \cap X_{2}$ y $Y=Y_{1} \cap Y_{2}$. Si $X \cap Y \neq \varnothing$, entonces $X_{1} \cap X_{2} \cap Y_{1} \cap Y_{2} \neq \varnothing$, con lo que $X_{1} \cap Y_{1} \neq \varnothing$ y $X_{2} \cap Y_{2} \neq \varnothing$, y, por tanto, $X_{1}=Y_{1}$ y $X_{2}=Y_{2}$ (pues $P$ y $Q$ son particiones), y, por consiguiente, $X=Y$. Luego si $X \neq Y$, entonces $X \cap Y=\varnothing$, que es la última que quedaba por demostrar.

La operación $\otimes$ de superposición es asociativa, conmutativa e idempotente, es decir, para cualesquiera particiones $P, Q, G$ del mismo dominio:

6.3. $(P \otimes Q) \otimes G=P \otimes(Q \otimes G)$

6.4. $P \otimes Q=Q \otimes P$

6.5. $P \otimes P=P$

El semigrupo $\left\langle\operatorname{Part}_{A}, \otimes\right\rangle$ tiene un clemento absorbente, $\mathrm{Sng}_{A}$, y un elemento neutro, $\{A\}$, es decir, para cada partición $P$ de $A$ : 


\section{6. $P \otimes \mathrm{Sgn}_{A}=\mathrm{Sng}_{A}$ \\ 6.7. $P \otimes\{A\}=P$}

¿Es la superposición de dos particiones siempre comparable con ellas respecto a finura? Sí. La superposición es siempre más o igual de fina que cada una de las particiones superpuestas. Para cualesquiera particiones, $P, \mathrm{Q}$, de $A$ :

\section{8. $P \otimes Q \leqslant P ; \quad P \otimes Q \leqslant Q$}

De aquí se sigue como corolario que la cardinalidad (el número de miembros) de la superposición es igual o mayor que la de las particiones superpuestas:

\section{9. $|\mathrm{P} \otimes \mathrm{Q}| \geqslant|\mathrm{P}| ;|\mathrm{P} \otimes \mathrm{Q}| \geqslant|\mathrm{Q}|$}

¿Es el semigrupo $\left(\right.$ Part $\left._{A}, \otimes\right)$ un grupo? No, no lo es. Si $A$ tiene al menos 2 clementos, entonces $\left(\operatorname{Part}_{A}, \otimes\right)$ no es un grupo, pues no puede haber una formación de inverso. En efecto, si $A$ tiene 2 o más elementos, siempre habrá particiones de $A$ que tengan 2 o más miembros. Sea $Q$ una de ellas. Si $\left\langle\operatorname{Part}_{A}, \otimes\right\rangle$ fuera un grupo, tendría que haber, un inverso de $Q$, es decir, una partición $Q^{\prime}$ de $A$, tal que $Q \otimes Q^{\prime}=\{A\}$. Pero entonces $2 \leqslant|Q| \leqslant\left|Q \otimes Q^{\prime}\right|$, por el corolario 6.9. Pero $\left|Q \otimes Q^{\prime}\right|=\{\{A\} \mid=1$. De donde se sigue que $2 \leqslant 1$. Esta contradicción muestra que no puede haber un inverso de $Q$ y que, por tanto, $\left(\operatorname{Part}_{\mathcal{A}}, \otimes\right)$ no es un grupo.

La superposición de dos particiones sólo es fecunda o interesante cuando ambas particiones superpuestas son incomparables. Sólo en ese caso se genera una partición nueva. Si las dos particiones superpuestas son comparables, su superposición se limita a reproducir la más fina de ellas. Sean $P, Q$ particiones de $A$.

\subsection{0. $P \leqslant Q \Rightarrow P \otimes Q=P$}

Prueba. Supongamos que $P \leqslant Q$. Sea $Z \in P \otimes Q$. Entonces (por 6.1.) hay un $X \in P$ y un $Y \in Q$, tales que $Z=X \cap Y$ y $Z \neq \varnothing$. Por 3.2. y puesto que $P \leqslant Q$, tenemos que $X \subset Y \vee X \cap Y \equiv \varnothing$. Pero $X \cap Y=Z \neq \varnothing$. Luego $X \subset Y$. Por tanto, $X \cap Y=X \mathrm{y}$, teniendo en cuenta que $Z=X \cap Y$, tenemos que $Z=X$. Luego $Z \in P$, pues $X \in P$. Así pues, $P \otimes Q \subset P$. Sea $Z \in P$. Entonces (por 3.1.) hay un
$Y \in Q$ tal que $Z \subset Y$. Luego $Z=Z \cap Y$ y, además, $Z \neq \varnothing$, ya que $Z \in P$ y $P$ es una partición. Luego $Z \in P \otimes Q$, por 6.1. Así pues, $P \subset P \otimes Q$. En resumen, $P \otimes Q=P$.

\section{FUSION DE PARTICIONES}

Sea $G$ un conjunto de subconjuntos de $A$, es decir, GCPP. Decimos que un individuo cualquiera $a \in A$ es $G$-conectable con otro individuo $b \in A$ si podemos pasar de un conjunto de $G$ que contiene $a$ a otro conjunto de $G$ que contiene $b$ a través de una serie de conjuntos no-disjuntos intermedios. En la figura siguiente los círculos representan conjuntos de $G$.

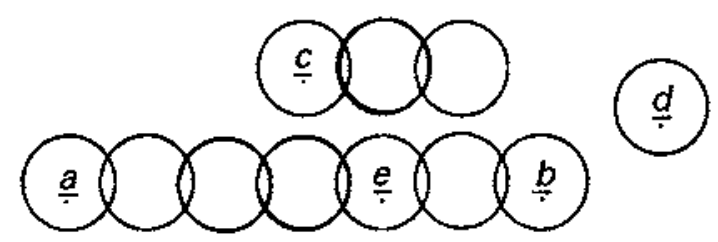

Mirando la figura diremos que a es conectable con $b$ y con $e$, pero no con $c$ o con $d$. Simbolicemos mediante $\mathrm{K}_{G}$ la relación de $G$-conectabilidad. Sea $G \subset \cup O P A$. Sean $a, b \in A$. Definimos:

$$
\begin{aligned}
\text { 7.1. } & a K_{G} b \Leftrightarrow \exists Z_{1} \ldots Z_{n} \in G \\
& \left(a \in Z_{1} \wedge b \in Z_{2} \wedge Z_{1} \cap Z_{2} \neq \varnothing \wedge \ldots\right. \\
& \left.\wedge Z_{n-1} \cap Z_{n} \neq \varnothing\right)
\end{aligned}
$$

Sea $G$ un recubrimiento de $A$. Está claro que la relación $K_{G}$ de $G$-conectabilidad es reflexiva, simétrica y transitiva en $A$. Para cualquier recubrimiento de $G$ de $A$ :

\section{2. $K_{G}$ es una relación de equivalencia en $A$.}

Consideremos ahora dos particiones cualesquiera de $A$, por ejemplo $P$ y $Q$. Evidentemente la unión $P \cup Q$ será un recubrimiento de $A$ y $K_{P \cup Q}$ será una relación de equivalencia en $A$. La relación $K_{P \cup Q}$ de $P \cup Q$-conectividad es la relación de equivalencia en que están dos elementos cualesquiera de $A$ si y sólo si es posible conectar el uno con el otro a través de una serie de taxones solapantes de entre los pertenecientes a cualquiera de las dos particiones $P \circ Q$. Como ya vimos, 
toda relación de equivalencia en $A$ induce una partición de $A$, a saber, el espacio cociente de $A$ por esa relación. Esto nos permite definir una nueva operación entre particiones, a la que $\mathrm{lla}_{\mathbf{a}}$ maremos fusión y simbolizaremos por $\oplus$. Sean $P$ y $Q$ particiones de $A$. Definimos:

$$
\text { 7.3. } P \oplus Q=A / K_{P \cup Q}=\left\{x_{K_{\text {PUQ }}} \mid x \in A\right\}
$$

De esta definición de $P \oplus Q$ como espacio cociente de $A$ por una relación de equivalencia se sigue por 2.6. que $\oplus$ es una operación que lleva de particiones de $A$ a particiones de $A$, es decir, que la fusión de dos particiones siempre es de nuevo una partición.

\section{4. $\oplus: \operatorname{Part}_{A} \times \operatorname{Part}_{A} \rightarrow \operatorname{Part}_{A}$}

Quizá no esté de más tratar de captar intuitivamente la diferencia entre la superposición y la fusión de particiones. Dadas dos particiones $P$ y $Q$ del mismo dominio $A$, su superposición $P \otimes Q$ es la partición que hace todas (y solas) las distinciones que hacen $A \circ B$, mientras que su fusión $P \oplus Q$ es la partición que hace sólo aquellas dis. tinciones comunes a $P$ y $Q$. El siguiente cuadro puede ayudar a visualizar estas operaciones. Se trata de partir el conjunto A de los puntos de la linea horizontal dibujada. Las particiones $P, Q$ $P \otimes Q$ y $P \oplus Q$ de $A$ se representan mediante filas de casillas.

La operación $\oplus$ de fusión es asociativa, con* mutativa e idempotente, es decir, para cualesquiera particiones $P, Q, G$ del mismo dominio:

\section{5. $(P \oplus Q) \oplus G=P \oplus(Q \oplus G)$}

7.6. $P \oplus Q=Q \oplus P$

7.7. $P \oplus P=P$
Como fácilmente se comprueba, cada una de las operaciones de superposición y fusión es absorbente respecto a la otra, es decir, para cualesquiera particiones $P$ y $Q$ del mismo dominio:

\section{8. $P \otimes(P \oplus Q)=P ; \quad P \oplus(P \otimes Q)=P$}

Hemos definido la operación de fusión de dos particiones de $A$ como el espacio cociente de $A$ por la relación de conectabilidad en la unión de ambas particiones. Ahora presentamos otra manera (equivalente) de definir la fusión de dos particiones.

Cuando fusionamos dos particiones, juntamos en un solo taxón todos los taxones de ambas par. ticiones que son comunicables entre si por un ca* mino de taxones no-disjuntos. La noción intuitiva de camino puede precisarse mediante una función numérica que oscila entre taxones comunicados (no-disjuntos) de ambas particiones. Dos taxones son comunicables si hay un camino de uno al otro. Un grupo máximo de taxones comunicables forman una isla. Y la fusión de ambas particiones es precisamente el conjunto de esas islas, que forman una nueva partición. Las siguientes definiciones precisan esta caracterización de la fusión.

Un camino en dos particiones $P$ y $Q$ es una función $f: \mathbb{N} \rightarrow P \cup Q$, tal que $f(n) \in P$ si $n$ es par, $f(n) \in Q$ si $n$ es impar, y para todo $n$ : $f(n) \cap f(n+1) \neq \varnothing$.

Un taxón $Z \in P \cup Q$ es comunicable (respecto a las particiones $P$ y $Q$ ) con otro taxón $W \in P \cup Q$ si y sólo si existe un camino $f$ en $P$ y $Q$, y números $n, m \in \mathbb{N}$, tales que $f(n)=Z$ y $f(m)=W$.

Dado un taxón $Y \in P \cup Q$, la isla de $Y$ (respecto a las particiones $P$ y $Q$ ) es la unión de todos los taxones comunicables con $Y$.

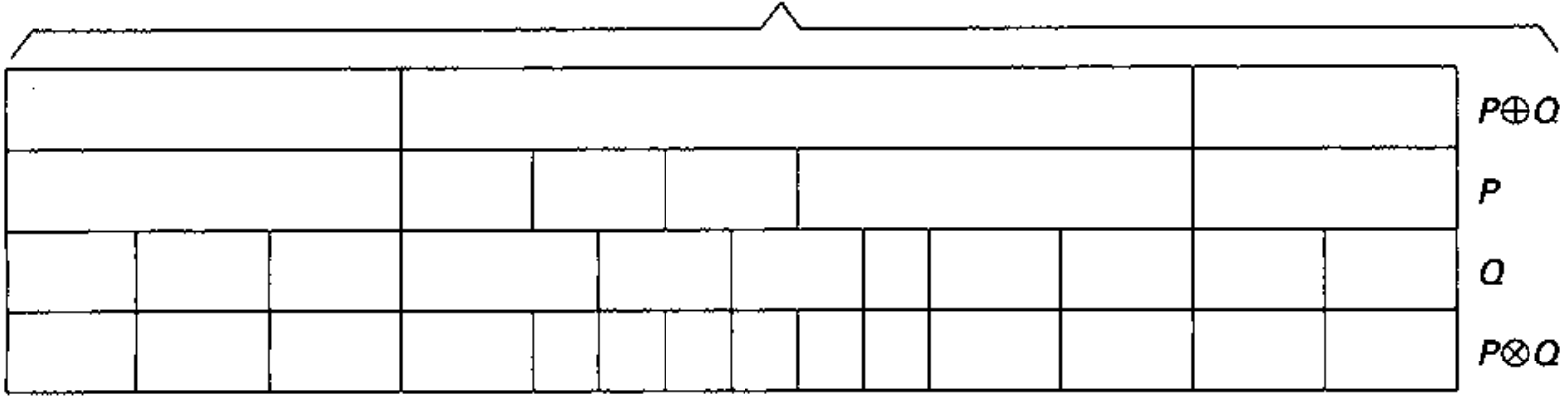


Isla $(Y)=\cup\{Z \mid Z \in P \cup Q \wedge Z$ es comunicable $\operatorname{con} Y\}$

Con esto estamos en posición de ofrecer la definición alternativa de la fusión. ${ }^{8}$ Para cualesquiera particiones $P, Q$ :

$P \oplus Q=\{\operatorname{Isla}(Z) \mid Z \in P \cup Q\}$

\section{EL RETICULO DE LAS PARTICIONES}

El hecho de que las operaciones $\oplus$ y $\otimes$ tengan las propiedades aquí señaladas indica que el conjunto de las particiones a $A$ constituye de algún modo un retículo. En efecto, así es. Ya habíamos visto que $\left(\operatorname{Part}_{A}, \leqslant\right.$ constituía una ordenación parcial. Un retículo es una ordenación parcial en la que cada par de elementos poseen un ínfimo (una máxima cota inferior) y un supremo (una mínima cota superior). Ahora bien, dadas dos particiones cualesquiera de $A$, por ejemplo $P$ y $Q$, su ínfimo es precisamente su superposición, $P \otimes Q$, y su supremo es precisamente su fusión, $P \oplus Q$. Por tanto, $\left\langle\operatorname{Part}_{A}, \leqslant, \otimes, \oplus\right\rangle$ constituye un reticulo. Probémoslo.

Como ya habíamos visto en 6.8., para cualesquiera particiones $P, Q$ de $A$ :

\section{1. $P \otimes Q \leqslant P ; \quad P \otimes Q \leqslant Q$}

Por tanto, $P \otimes Q$ es una cota inferior de $P$ y $Q$. Para demostrar que es el ínfimo de $P$ y $Q$ aún nos queda por probar que $P \otimes Q$ es la máxima de sus cotas inferiores. Scan $P$ y $Q$ particiones de $A$ :

\section{2. $\vee G \in \operatorname{Part}_{A}(G \leqslant P \wedge G \leqslant Q \Rightarrow G \leqslant P \otimes Q)$}

Prueba. Sea $G$ una partición de $A$, tal que $G \leqslant P$ y $G \leqslant Q$. Tenemos que probar ahora que $G \leqslant P \otimes Q$, es decir, por 3.1., que para cada $X \in G$ hay un $Z \in P \otimes Q$, tal que $X \subset Z$. Sea $X \in G$. Puesto que $G \leqslant P$ y $G \leqslant Q$, habrá un $Y \in P$ y un $W \in Q$, tales que $X \subset Y$ y $X \subset W$. Por tanto, $X \subset Y \cap W$. Además, $Y \cap W \neq \varnothing$, pues $X \neq \varnothing$, ya que es un taxón de una partición. Luego hay un $Z$, a saber $Z=Y \cap W$, tal que $Z \in P \otimes Q$, por 6.1., y $X \subset Z$.

Del 8.1. y 8.2. se sigue:

$$
\text { 8.3. inf }\{P, Q\}=P \otimes Q
$$

La fusión de dos particiones es una cota supe- rior de ambas particiones. Para cualesquicra particiones, $P, Q$ del mismo dominio $A$ :

\section{4. $P \leqslant P \oplus Q ; \quad Q \leqslant P \oplus Q$}

Prueba. Sea $X \in P$ un taxón cualquiera de $P$. Puesto que $X \neq \varnothing$, hay al menos un elemento en $X$; llamémosle $a: a \in X$. Sea $x$ un elemento cualquiera de $X$. Entonces $x K_{P \cup Q} a$, por la definición 7.1. Por tanto, $x \in a_{K_{\text {plio }}}$. Así pues, para cualquier $x \in A$ ocurre que $x \in a_{K_{\text {plo }} \text {, }}$ es decir, $X \subset a_{K_{p \cup Q}}$, donde $a_{K_{p \cup Q}} \in P \oplus Q$, por 7.3. En resumen, para cada taxón $X \in P$ hay un taxón $Y \in P \oplus Q$ (a saber, $a_{K_{\text {m: }}}$ ), tal que $X \subset Y$ y, por consiguiente, $P \leqslant P \oplus Q$. De igual modo se prueba $Q \leqslant P \oplus Q$.

Una vez probado que $P \oplus Q$ es una cota superior de $P$ y de $Q$, para demostrar que es el supremo de $P$ y $Q$ nos queda por probar que $P \oplus Q$ es la mínima de las cotas superiores de $P$ y $Q$. Sean $P$ y $Q$ particiones de $A$.

\section{5. $\vee G \in \operatorname{Part}_{A}(P \leqslant G \wedge Q \leqslant G \Rightarrow P \oplus Q \leqslant G)$}

Prueba. Sea $G$ una partición de $A$, tal que $P \leqslant G$ y $Q \leqslant G$. Tenemos que probar que $P \oplus Q \leqslant G$, es decir, que para cada $X \in P \oplus Q$ hay un $W \in G$, tal que $X \subset W$. Sea $X \in P \oplus Q . X \neq \varnothing$, pues $X$ es un taxón, y por tanto tendrá al menos un miembro $a \in X$. Puesto que $P$ es una partición de $A$ y $a \in A$, para algún $Y \in P$ ocurre que $a \in Y$. Puesto que $P \leqslant G$, hay un $W \in G$, tal que $Y \subset W y$, por tanto $a \in W$. Este es el $W$ que buscábamos. Hemos de probar que $X \subset W$. Sca $x \in X$. Hemos de probar que $x \in W$. Puesto que $P \oplus Q$ es un conjunto de clases de equivalencia, por 7.3., y $a \in X \in Q \oplus Q$, resulta que $X=a_{K_{p, c}}$. Puesto que $x \in X$, ocurre que $a K_{P \cup Q} x$. Por tanto, hay taxones $Z_{1} \ldots Z_{n} \in P \cup Q$ tales que $a \in Z_{1} \wedge x \in Z_{\mathrm{n}} \wedge$ $Z_{1} \cap Z_{2} \neq \varnothing \wedge \ldots \wedge Z_{n-1} \cap Z_{n} \neq \varnothing$, según la definición 7.1. Puesto que $P \leqslant G$ y $Q \leqslant G$, cada taxón de $P$ o de $Q$ está incluido cn un taxón de $G$, por lo que habrá $V_{1}, \ldots V_{\mathrm{n}} \in G$, tales que $Z_{\mathrm{i}} \subset V_{\mathrm{i}} \quad($ para $1 \leqslant \mathrm{i} \leqslant \mathrm{n})$. Como $Z_{1} \cap Z_{2} \neq \varnothing$, $V_{1} \cap V_{2}=\varnothing y$, por tanto, $V_{1}=V_{2}$, pues $G$ es una partición (y si $V_{1} \neq V_{2}$, entonces $V_{1} \cap V_{2}=$ $\varnothing)$. De igual modo: $V_{2}=V_{3}, \ldots V_{n-1}=V_{n}$. Puesto que $a \in W$ y $a \in V_{1}$ (pues $a \in Z_{1}$ y $Z_{1} \subset V_{1}$ ), y tanto $W$ como $V_{1}$ son taxones de la partición $G, W=V_{1}$. Así pues, $W=V_{1}=$ $V_{2}=\ldots=V_{n}$. Puesto que $x \in Z_{n}, Z_{n} \subset V_{n}$ y 
$V_{\mathrm{n}}=W$, resulta que $x \in W$. Por consiguiente $X \subset W$, que es lo que había que probar. Luego $P \oplus Q \leqslant G$.

De 8.4. y 8.5. se sigue:

\section{6. $\sup \{P, Q\}=P \oplus Q$}

De 8.3. y 8.6., y del hecho de que $\left\langle\operatorname{Part}_{A} \leqslant\right.$, es una ordenación parcial, se sigue que las particiones de un dominio dado $A$ forman un retículo respecto a las operaciones de superposición y de fusión. Para cualquier $A$ :

\section{7. $\left\langle\operatorname{Part}_{A}, \leqslant, \otimes, \oplus\right\rangle$ es un retículo}

Un retículo es complementario si para cada uno de sus elementos hay al menos un complemento, es decir, otro elemento tal que el ínfimo de ambos es el elemento mínimo de la ordena* ción parcial correspondiente y el supremo de ambos es el elemento máximo. En el caso particular del retículo de las particiones de $A$, un complemento de un elemento $a \in A$ es otro elemento $b \in A$, tal que $a \otimes b=$ Sng $_{A}$ y $a \oplus b=\{A\}$. De hecho cada partición de $A$ tiene al menos un complemento, aunque no vamos a exponer aquí la prueba, que es un poco complicada. Pero anotamos que:

\section{8. $\left\langle\operatorname{Part}_{A}, \leqslant, \otimes, \oplus\right\rangle$ es un retículo complementario}

Un álgebra de Boole es un retículo complementario y distributivo. ¿Es el retículo de las particiones de $A$ un álgebra de Boole? No, no lo es, pues no es distributivo. Si fuera distributivo, valdría que para cualquiera particiones $P, Q, G: P \otimes(Q \oplus G)=(P \otimes Q) \oplus(P \otimes G)$. Consideremos ahora un contraejemplo. Sea $A=\{0,1,2\}, P=\{\{0\},\{1,2\}\}, Q=\{\{0,1\}$, $\{2\}\}, G=\{\{0,2\},\{1\}\}$.

Resulta que

$P \otimes(Q \oplus G)=P \otimes\{A\}=P$ $(P \otimes Q) \oplus(P \otimes G)=$ Sng $_{A} \oplus$ Sng $_{A}=$ Sng $_{A}$

$$
\begin{aligned}
\text { Pero Sng } & =\{\{0\},\{1\},\{2\}\} \neq \\
\{\{0\},\{1,2\}\} & =P
\end{aligned}
$$

Por tanto, el retículo no es distributivo.

En un retículo distributivo cada elemento tiene a lo sumo un complemento. Pero puesto que el retículo de las particiones no es distributivo, una partición puede tener más de un complemento. $\mathrm{Y}$, en efecto, así es. El mismo contraejemplo que acabamos de considerar ejemplifica también esta multiplicidad de complementos, pues tanto $Q$ como $G$ son complementos de $P$, como fácilmente se comprueba.

\section{NOTAS}

1 Según la ya clásica definición de G. Simpson en Principles of Animal Taxonomy (Columbia University Press, New York 1961), p. 11, "taxonomía es el estudio teórico de la clasificación, incluyendo sus bases, principios, procedimientos y reglas". Aquí entendemos por $t a-$ xonomía formal la parte más abstracta de la taxonomía, que sc limita a considerar y explicitar las estructuras formales o matemáticas implícitas en la actividad de clasificar.

${ }^{2}$ La mayoría de los biólogos sistemáticos usan el tér * mino «identificación" para este proceso, pero esa terminología puede ser confundente en estudios formales. Por eso preferimos aquí el término "diagnóstico" utilizado, por ejemplo, en N. JARDINE y R. SrBSON: Mathematical Taxonomy (John Wiley. London 1971), p. 267.

${ }^{3}$ JoHN R. GreGG: The Language of Taxonomy, $\mathrm{Co}-$ lombia University Press, New York, 1954.
4 A. F. PARKER-RHodes: Review of The Languaje of Taxonomy. Pbilosophical Review 66, 1957.

5 ABE SKLAR: "On Category Overlapping in Taxonomy". Incluido en From and Strategy in Science (edited by J. R. Gregg y F. T. Harris). Reidel Publishing Co. Dordrecht 1964.

6 Roger C. Buck y David L. Huld: "The Logical Structure of Linnaean Hierarchy», Systematic Zoology, $15,1966$.

Nrcholas Jardine, "A Logical Basis for Biological Classification", Systematic Zoology, 18, 1969.

8 La Introducción de la noción de fusión de particiones y las dos definiciones (la 7.3 y ésta) de esta operación aquí presentadas se deben a Enrique Casanovas. 